\title{
The Optimized Analysis on the Process of Project Management
}

\author{
Yang Anrong, a \\ ${ }^{1}$ School of Management Huazhong University of Science and Technology Wuhan430074, China \\ a491630736@qq.com
}

Keywords: project management, work-flow, model, PSO control algorithm

\begin{abstract}
The paper is about the construction of engineering process management mechanism and framework based on work-flow, and of the overall framework model including: project management process, organization, and information integration. Besides, PSO algorithm is improved, and the model on it is also analyzed. PSO is an algorithm with better research performance and more convenient practical manipulation, and is more suitable for the solution of the model of the paper. The project schedule and cost is well controlled and managed by the algorithm.
\end{abstract}

\section{Introduction}

The optimization of the project is not a new problem, but it is still a problem to be studied ${ }^{[1,2]}$. The existing optimization method is very limited in its ability to solve practical engineering optimization problems $^{[3,4]}$, which need to be integrated to improve the optimization effect. In recent years, as logistics engineering has gradually become the hot competition of logistics market, the company and other COSCO Logistics subsidiary companies develop their own project management personnel by successively sending their personnel to participate in the project - East Electric Model - for independent development of their logistics engineering. But, because the majority of its employees have no experience of logistics engineering, staff in the process of practice is also difficult to fully understand the overall management process of a project.

\section{The analysis on the process management mechanism and framework of the company}

The integrated management of engineering project group is dependent on the coordination of the above three aspects: the organization structure, process management and information technology. Only when integration is carried out on three levels:the process, the organization and the information, can the project group be successful.

Integrated information management system and the ring network organization structure based on IPT are dedicated to realize information integration across geographical boundaries and organizational boundaries. Information management system is from the point of view of information technology, and the ring network organization structure of the IPT is from the perspective of organization. Information integration can promote the flattening of the organizational structure of the project group, and the integration is very important for the effective solution of the problem of information exchange in engineering projects. At the same time, the establishment of the organization can ensure the barrier-free accessibility of information center. Process integration is realized based on information integration and organization integration. Information integration and organization integration provides technology and organization platform for process integration. Process integration is a higher level above information integration and organization integration. Efficient process integration can bridge between the organization integration and information integration. The integration of organization, information and process promotes the realization of the integrated management of engineering project group. 


\section{Optimized design on the engineering project management process}

\section{Project management based on work-flow technology}

The work-flow management system includes three functions: the establishment, operation and interaction of the work-flow. The work-flow is established by using work-flow modeling tool, and each the process is defined and organized. The core part of the whole work-flow is the phase of operation, which defines the resource information for each activity allocation through setting the required parameters, is responsible for creating, deleting the entire process control, completes the transfer between activities, and monitors the activities of the whole process. Interactive stage is the ability to achieve human-computer interaction, an interaction between user and program of different task correlation. Work-flow architecture is shown in Figure 1:

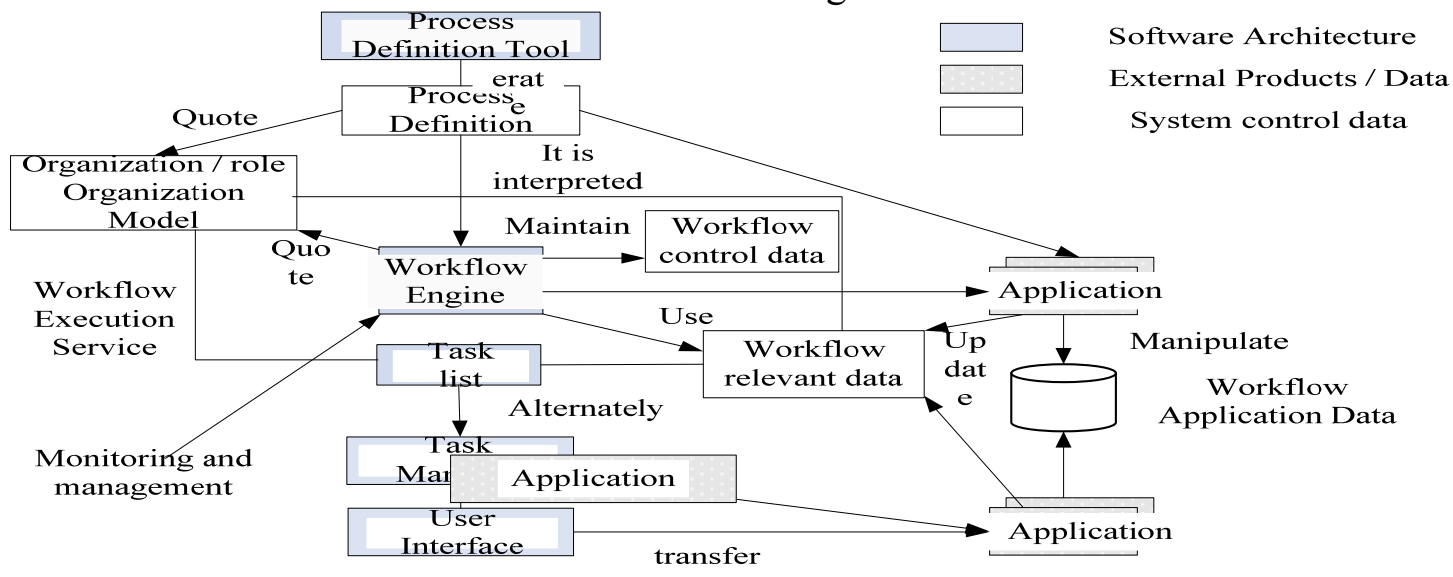

Figure 1work-flow architecture

The core of the work-flow is work-flow engine, which is responsible for the definition of the work-flow process, creating the initialization process instance, controlling the flow direction, recording the process flow, and terminating the running process. The whole process is effectively controlled by work-flow engine. In the process control, activity refers to a logical step to complete the work-flow, and the main attributes include the activity name, activity type, pre/post activity conditions, other scheduling constraints. The work-flow management coalition will device activities into tasks, rules, sub processes and rules, through which to achieve the control of the whole process. As is shown in Figure 2, multi-project group is also a project group, both of which are the complex integrated system that is composed of the interconnected individuals, and management of these two systems can result in a similar scale benefit. But the multi- project group is more complex than the project group, the more individual levels a multi-project group contains, the more complex the structure within it is, which will be further studied in the future.

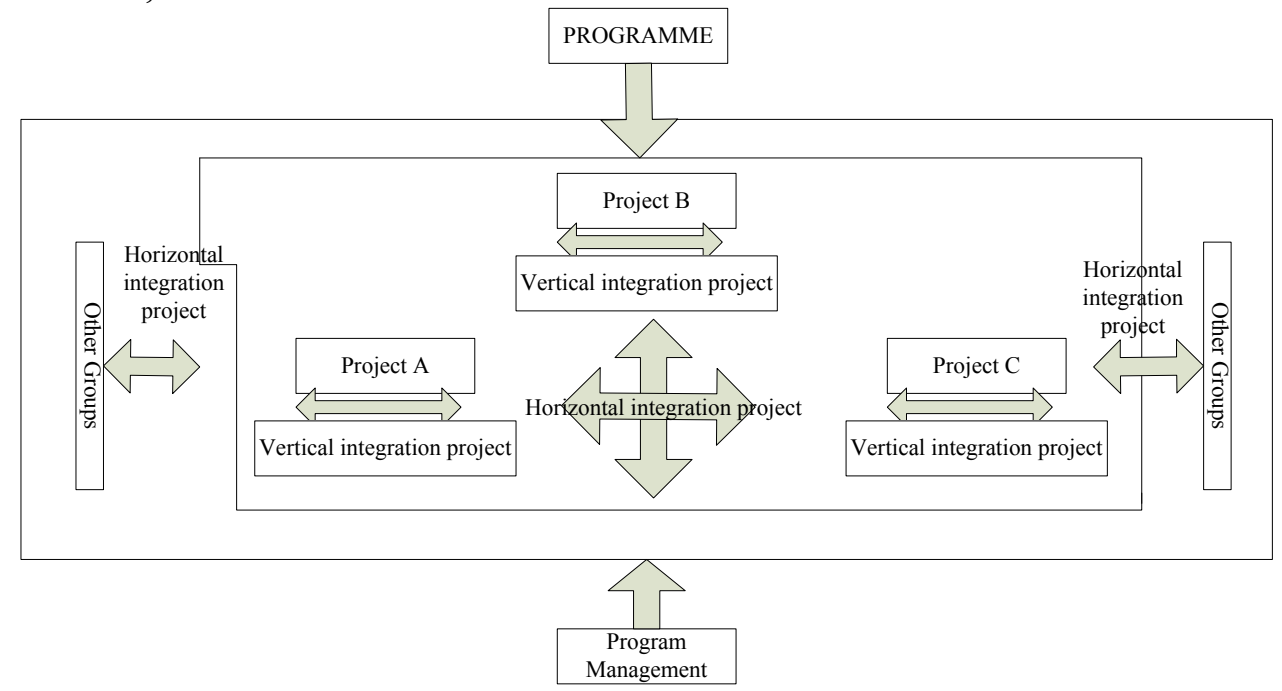

Figure 2 multi-level integrated management of engineering projects 


\section{The optimization of Engineering project process based on particle algorithm}

Particle swarm optimization (PSO) is a model for simulating the behavior of birds. PSO algorithm firstly assumes that in the target search space of a $\mathrm{D}$ dimension, there are $\mathrm{m}$ particles to form a community, where the first $\mathrm{i}$ particle represents a $\mathrm{D}$ dimensional vector $\overline{x_{i}}=\left(x_{i 1}, x_{i 2}, \ldots, x_{i n}\right), \mathrm{i}=1$, $2, \ldots, \mathrm{m}$, that is, the position of the i particles in the $\mathrm{D}$ dimensional search space is $\overline{x_{i}}$. In other words, the position of each particle is a potential solution. By applying it to an objective function to calculate the fitness value, which is measured by the fitness value. The flying speed of the first i particle is also a vector of $\mathrm{D}$ dimension, that is $\overline{v_{i}}=\left(v_{i 1}, v_{i 2}, \ldots, v_{i n}\right), \mathrm{i}=1,2, \cdots, \mathrm{m}$. If the best position searched to date of the I particle is $\overline{p_{i}}=\left(p_{i 1}, p_{i 2}, \ldots, p_{i n}\right), \mathrm{i}=1,2, \ldots, \mathrm{M}$, the best position searched to date of the whole particle swarm is $\overline{p_{g}}=\left(p_{g 1}, p_{g 2}, \ldots, p_{g n}\right), \mathrm{g}=1,2, \cdots, \mathrm{m}$.

PSO algorithm uses the following formula for particle operation. The speed and new position of particle will be updated according to the following formula:

$$
V_{i d}=V_{i d}+c_{1} \operatorname{rand}\left(P_{i d}-X_{i d}\right)+c_{2} \operatorname{rand}\left(P_{g d}-X_{i d}\right)
$$

In the formula: $c_{1}, c_{2}$ is constants acceleration, usually as $c_{1}=c_{2}=2$. Rand() and Rand () is the random function, with range $[0,1]$. Vi is particle velocity, and $V_{i} \leq V_{\max }$ (Set the maximum speed, determine the maximum movement distance of particles in a circle, usually set to the range of the particle), if $V_{i} \geq V_{\text {max }, d}$, then $V_{i d}=V_{\text {max }, d}$.

The process description of the basic particle swarm algorithm is as follows:

(1) In the D dimension problem space, a group of particles with a size of $\mathrm{M}$ is initialized;

(2) For each particle, the fitness function value is calculated by using its position (D dimensional variable); compare its current fitness value with the best value pbest it experiences, if it works well, apply it to pbest. The position of the pbest (D dimensional variable) is also equal to the current position;

(3) Compare the current fitness value of all particles with the best value pbest the population experiences, if it works well, apply it to pbest. The location of the gbest ( $\mathrm{D}$ dimensional variable) is also equal to the current best gbest position;

(4) The velocity and position of the particle is updated according to the formula (4-1) and (4-2);

(5) If the end condition is not reached (usually for the preset computation accuracy or the maximum number of iterations), return 2

(6) If the end condition is not reached, end.

Definition 1 (global minimum): a function is given $f: \Omega \subseteq R^{n} \rightarrow R, \Omega \neq \phi, f^{\prime \prime}=f() \overline{x^{*}}>-\propto$ is called global minimum as for the value of $\bar{x} \in \Omega$. If the condition is set as $\forall \bar{x} \in \Omega: f\left(\overline{x^{*}}\right) \leq f(\bar{x})$, $x^{*}$ is the global minimum, $\mathrm{f}$ is objective function, $\Omega$ is feasible region $(\Omega \in \mathrm{S})$, of which $\mathrm{S}$ represents the whole research space.

Definition 2 (general multi-objective optimization ): as looking for the vector $\overline{x^{*}}=\left({\overline{x_{1}}}^{*},{\overline{x_{2}}}^{*}, \ldots,{\overline{x_{j l}}}^{*},\right)$, meet the $\mathrm{m}$ inequality constraints $\mathrm{g}_{\mathrm{j}}(\bar{x})>0, \mathrm{i}=1,2, \ldots, \mathrm{m}$, as well as the $\mathrm{P}$ equation limit $\mathrm{h}_{\mathrm{j}}(\bar{x})>0, \mathrm{i}=1,2, \ldots, \mathrm{p}$, at the same time, make the vector function $\vec{f}(\vec{x})=\left[f_{1}(\vec{x}), f_{2}(\vec{x}), \ldots, f_{k}(\vec{x})\right]^{T}$ reach the optimal, of which $\vec{x}=\left(\overrightarrow{x_{1}}, \overrightarrow{x_{2}}, \ldots, \overrightarrow{x_{n}}\right)^{T}$ is the vector of decision variables.

If there is unfeasible vector that weakens some standards while not leading to at least one standard within them being strengthened, this is defined as arrangement optimization. Unless otherwise specified, permutation optimal will be considered as the mean number of the whole decision variable space. 
Definition 3 (permutation optimal): vector $\vec{u}=\left(u_{1}, u_{2}, \ldots, u_{n}\right)^{T}$, known to have the advantage (shown as $\vec{u} \leq \vec{v})$ )over $\vec{v}=\left(v_{1}, v_{2}, \ldots, u_{k}\right)$, only when $u$ part is less than $\mathrm{v}$, for instance:

$\forall i \in\{1,2, \ldots, k\}, u_{i} \leq v_{i} \wedge \exists i \in\{1,2, \ldots, k\}, u_{i} \leq v_{i}$

Definition 5 (permutation optimal set): for a MOP $\mathrm{f}(\mathrm{x})$, the permutation optimal set $\left(\mathrm{P}^{*}\right)$ is defined as:

$$
P^{*}:=\left\{\vec{x} \in \Omega \mid \exists x^{1} \in \Omega \cdot \vec{f}\left(x^{1}\right) \leq \vec{f}(x)\right.
$$

Definition 6 (permutation front): for a given MOP $\mathrm{f}(\mathrm{x})$ and permutation optimal set $\left(\mathrm{P}^{*}\right)$, permutation front is defined as:

$$
P F^{*}:=\left\{\vec{u}=\vec{f}=\left(f_{1}(x), \ldots, f_{k}(x) \mid x \in P^{*}\right\}\right.
$$

In general, it is not possible to find an analytical linear expression or surface expression that contains these points. The general procedure that generates the arrangement of the front, that is, the calculation of the feasible region $\Omega$ and its corresponding $\mathrm{f}(\Omega)$. When there is plenty of data, it is possible to determine the non dominant points and generate permutation front. The results of permutation optimal is called a non secondary, admissible or effective result, and the corresponding vector is called a non dominant vector.

The process of multi-objective particle swarm optimization(MOPSO)is as follows:

(1) Initialize the target group POP:

For $\mathrm{i}=$ from 0 to $\mathrm{MAX} / * \mathrm{MAX}=$ number of particle*/ $\mathrm{i}$

Initialize POP[i]

(2) Initialize the speed of each particle:

(1)For $i=$ from 0 to MAX (2)VEL[i $]=0$

(3) The evaluation of each particle in the group (POP).

(4) The location of the non dominant vector particles in the storage warehouse REP.

(5) Generate the super cube of the research space currently being explored to pace particles. Take these super cube for a corresponding system, in which the corresponding position of each particle is determined according to the value of the objective function.

(6) Initialize the memory of each particle(This memory, as a guide across the research space, will also be stored in the warehouse.):

(1)For $i=$ from 0 to MAX (2)PBESTS [i ] =POP[i]

(7) When the maximum number of cycles is still not found:

(1) The following formula is used to calculate the speed of each initialized particle:

$V E L[i]=W \times V E L[i]+R_{i} \times\left(P B E S Y[i]-P O P[i]+R_{2} \times(R E P[h]-P O P[i])\right.$

(2) The new position of the particles is calculated by the following method:

$$
\text { POP }[\mathrm{i}]=\mathrm{POP}[\mathrm{i}]+\mathrm{VEL}[\mathrm{i}]
$$

(3)Keep the particles in the research space, in case of escaping from the boundary(avoid the result of an ineffective search range). When a decision variable is out of its borders, the following two steps should be carried out: in the first step, the decision variables are taken from the value of the corresponding boundary (at the top or the bottom). The second step, its speed multiplied by -1 , so that it is to search the opposite direction.

(4)Find the value of each particle in POP

(5) Update the contents of the REP and the location of the particles in the hypercube. This update is mainly to put all the vector currently in the non-dominant position into the warehouse. Eliminate any advantage from the warehouse in the process. Due to the limitation of warehouse capacity, when it is full, it will be kept according to secondary standard: particles of small population area in the target space is given more priority over those of higher population area.

(6) When the particle's present position is better than that in the memory, the following formula is used to update the position of the particles. The standard of the memory location that is reserved for PBESTS [i ] = POP [i] is similar to the use of permutation(for example: if the current position is occupied by the memory location, the memory location is retained; otherwise, the memory location is 
replaced by the current location, if they are not occupied, but occupied by others, then randomly choose one of them.

(7) Increase the frequency of cycles.

(8) End

\section{Experimental verification and simulation}

In this paper, the case study is about a project of a staff healing center in Tianjin. Most of the buildings are two-stories high, some are one-story high and some six-stories high, building height is designed according to the functional space requirement, the height of the whole building group is less than 24 meters. For the construction unit, the construction process should be optimized in all aspects, including the optimization of procedure, multi-object integration and performance. The learning factor for this case is $\mathrm{C} 1=\mathrm{C} 2=0.5$, the inertia weight is reduced from 0.9 to 0.4 , Vmax is the width of the search space.

Table 1 simulation of the optimized results

\begin{tabular}{|c|c|c|c|}
\hline Operation code & Duration (days) & Cost value (wan) & Reliability \\
\hline $\mathrm{A}$ & 42 & 3681 & 0.87 \\
\hline B & 154 & 4069 & 0.94 \\
\hline $\mathrm{C}$ & 112 & 2474 & 0.96 \\
\hline $\mathrm{D}$ & 56 & 1243 & 0.90 \\
\hline $\mathrm{E}$ & 210 & 8031 & 0.98 \\
\hline $\mathrm{F}$ & 196 & 6093 & 0.91 \\
\hline G & 182 & 5772 & 0.97 \\
\hline $\mathrm{H}$ & 168 & 3079 & 0.87 \\
\hline $\mathrm{I}$ & 70 & 1287 & 0.89 \\
\hline
\end{tabular}

Applying MATLAB programming to run 1000 times, the optimization results are shown in table 1. At the same time, the calculation period of the project is 546 days, which is less than the contract period of 630 days; the total cost for the construction under the calculation period is 11810000 yuan, the service cost is 369100000 , and the total cost of the system is $357290000 y u a n$, which is lower than the expected total cost of the project; service satisfaction was 93.4\%; reachable quality grade is 0.9211; meet the requirements of the quality level of engineering target, and control the cost by using these optimization value to make the progress control, cost control, quality control and service control well integrated

\section{Summary}

The optimization of the project is not a new problem, But so far, it is a question that needs to be further studied.There are many studies on the optimization of engineering project nowadays, but the existing optimization method is much limited by the ability to solve practical engineering problems. In this paper, a general framework model of integrated management of engineering project is constructed. By applying integrated management theory, starting from the integration level of the project group, the connotation and mechanism of the project group management process, management organization form and information integration is expounded to construct the general framework model of project management process, organization, information integration. After the analysis of the model by using PSO algorithm, the proposed algorithm can have a better control and management of the project schedule and cost.

\section{References}

[1] Guo Xi-wen \& Chen Xiao-hong,Development Trend of Engineering Logistics and Its Countermeasures Analysis, Finance and Economy, Vol 22,2008,86-87

[2] Zhang Xiao-yan, Approach to Improve the Logistics Management of Engineering Projects in China,,Science and Technology Wind,Vol 8, 2010, 42

[3] Zhu Mei-hong \& Xu Li-juan, Optimization Strategy of Logistics Management in Water Conservancy and Hydropower Projects, Intelligence, Vol 33, 2009, 252-253 
[4] Liang Shui-kuang, A survey on the development trend of domestic project management, Inspection and Cost of Construction Supervision, Vol 10, 2008, 47-50

[5] Yu Xiao-xian \& Zhou Ling-yun, Research on the Introduction of Project Management into Logistics Enterprises,Shopping malls modernization,Vol 1, 2007, 145-147

[6] Ding Rong-gui \& Yang Nai-ding, Project organization and team, Beijing, Machinery Industry Press, 2011, 4-5

[7] Jiao Zhi-qi, Thinking on the Process Management of Construction Project, China Construction Information, Vol4, 2009, 119-121. 